\title{
Design of a 5 GHz Reflectarray with Reduced Size Unit Cell and Extremely Low Phase Sensitivity
}

\author{
Arnab PATTANAYAK ${ }^{1}$, Goutam RANA ${ }^{2}$, Siddhartha P. DUTTAGUPTA ${ }^{1,2}$, \\ Prasanna S. GANDHI ${ }^{1,3}$, Amit A. DESHMUKH ${ }^{4}$ \\ ${ }^{1}$ CRNTS, IIT Bombay, Mumbai- 400076, India \\ ${ }^{2}$ Dept. of Electrical Engineering, IIT Bombay, Mumbai- 400076, India \\ ${ }^{3}$ Dept. of Mechanical Engineering, IIT Bombay, Mumbai- 400076, India \\ ${ }^{4}$ Dept. of Electronics and Telecommunication Engineering, DJSCE, Mumbai- 400056, India
}

arnab_pattanayak@iitb.ac.in, \{goutam_rana, sdgupta, gandhi\}@iitb.ac.in, amitdeshmukh76@rediffmail.com

Submitted November 23, 2017 / Accepted April 27, 2018

\begin{abstract}
This article presents a novel, compact reflectarray antenna operating at $5 \mathrm{GHz}$. The array consists of two types of phasing element - square ring and complementary square ring. The complementary square ring is used for the phase values that are not covered by the single ring element. The grid size of the array is $0.28 \lambda \times 0.28 \lambda$ at operating frequency $5 \mathrm{GHz}$ that is much smaller than the conventional periodicity $0.5 \lambda \times 0.5 \lambda$. The aim of unit cell design is to have a slower slope of the reflection phase graph without sacrificing the phase range of $360^{\circ}$ where in general there is a trade-off between these two goals. The maximum slope in reflection phase graph obtained here is $34 \% \mathrm{~mm}$. The proposed array is fabricated on a low loss PTFE substrate of thickness $3.175 \mathrm{~mm}(0.053 \lambda$ at $5 \mathrm{GHz})$ and illuminated by a horn antenna. Radiation pattern results show a very precise far-field beam with 3-dB beamwidth of $7^{\circ}$ and $7.3^{\circ}$ for two principal planes respectively. The gain of the antenna is $26 \mathrm{dBi}$ at $5 \mathrm{GHz}$.
\end{abstract}

\section{Keywords}

Reflectarray, phase sensitivity, single ring, complementary single ring

\section{Introduction}

The microstrip reflectarray puts together the notions of parabolic reflector and planer phased array antenna. There are certain distinct advantages of using the reflectarray compared to its parabolic counterpart, such as: lightweight, low cost, easy to fabricate, suitable for batch fabrications, easy to mount, etc. [1], [2]. The reflectarray consists of isolated phasing elements that are illuminated by a feed antenna. Therefore, they do not need the complex and bulky feeding network [3]. The phasing elements are pre-designed with a particular phase compensation to reradiate the incident field and form the engineered wavefront in the far-field. This required phase compensation is obtained from reflection phase graph that is generated from varying one of the geometrical parameters of the microstrip phasing element [4], [5]. Two main characteristics of this reflection phase graph are important for designing a phasing element: one is phase range and another one is gradient (slope) of the phase graph. Inadequate phase range and steeper slope compared to manufacturing tolerance introduce phase error that in turn degrades the radiation performance of reflectarray. However, it is difficult to achieve both $360^{\circ}$ phase range and reasonably slower slope of reflection phase graph simultaneously. In order to achieve these both goals together several phasing elements are reported in recent years such as: multi-layered structures [6], single layered multi-resonant structures [7-9] and shorted patch with a foam placed underneath it [10]. Ruyuan Deng et al. used both multi-layered and multiresonant phoenix type element to design a reflectarray for quad-band application at $\mathrm{Ku}$ and $\mathrm{Ka}$ bands [11]. Most of these designs involve either multi-layer fabrication process or thick substrate with extra foam layer that increases manufacturing and material costs. Moreover, stacked designs need proper alignment between subsequent layers. Apart from these approaches, another most promising solution is reported in [12], where single ring is employed as the phasing element. In that work, in addition to conventional methods (fixed width method and fixed ratio method) two new design methods are introduced to reduce phase sensitivity without scarifying phase range: one is increasing the outer length and decreasing the inner length simultaneously and another one is increasing the inner length with fixed outer length. In [13] D. Cadoret et al. varied both length of the patch and that of crossed slots to get reflection phase graph for a dual polarized reflectarray. However, all these reported phasing elements have the grid spacing of the order of $\lambda / 2$. Moreover, these reflectarrays are designed to operate at or above $10 \mathrm{GHz}$. Towards lower frequency of operation (below $10 \mathrm{GHz}$ ), compactness is an important issue because unit cell size is inversely proportional to the operating frequency. Furthermore, the smaller grid size with higher phase accuracy of each grid would give a better realization of parabolic phase distribution that is required for highly directive emission of the reflectarray [14]. Pre- 
viously, in the reports reduced size phasing elements are employed for the purpose to get broadband reflectarray, although these subwavelength unit cells often fail to attain the full phase range due to etching tolerance on small gapsize [15-17]. In our previous article, we designed a reflectarray, where full $360^{\circ}$ phase range was achieved with three different types of subwavelength phasing element [18]. However, in that paper we did not conduct any study about phase sensitivity of reflection phase graph as well as broadband nature of the reflectarray antenna.

In this paper, to keep the overall size of the reflectarray compact the unit cell size is taken as $0.28 \lambda \times 0.28 \lambda$ at operating frequency $5 \mathrm{GHz}$. This is much smaller than the conventionally designed near-resonant $(0.5 \lambda)$ variable sized patches. We first choose the single square ring as phasing element. The reduction in slope of the reflection phase graph is attributed to the inner length variation of ring element while fixing the outer length. This procedure of varying inner length is repeated for all the higher successive values of outer length. This procedure covers a significant phase angle range of around $316^{\circ}$. The rest phase range $\left(\sim 44^{\circ}\right)$ is covered by introducing the second type of phasing element, which is complementary square ring. Therefore, full $360^{\circ}$ reflection phase range is achieved with a very gentler slope of reflection phase graph using reduced size unit cells $(0.28 \lambda \times 0.28 \lambda)$ at operating frequency $5 \mathrm{GHz}$. Moreover, the subwavelength size unit cell helps to provide a broad gain-bandwidth.

\section{Phasing Element Design}

\subsection{Single Square Ring}

Single square ring is a popular phasing element due to its simple shape [12]. In general, this element has two degrees of freedom to generate reflection phase graph. The first one is its outer length for a particular width i.e. overall size and the second one is its width [4]. The schematic of a single square ring is shown in Fig. 1(a). FEM based frequency domain solver in CST MWS is used to calculate reflection phase of the phasing element. Unit-cell boundary conditions are applied in transverse $\mathrm{x}$ and $\mathrm{y}$ directions and Floquet port boundary conditions are applied in positive and negative z-directions as shown in Fig. 1(b). An xpolarized TEM plane wave impinges on the phasing element. Phasing elements are considered on PTFE substrate $\left(\varepsilon_{\mathrm{r}}=2.2\right.$ and $\left.\tan \delta=0.0005\right)$ of thickness $3.175 \mathrm{~mm}$ that is used to fabricate the full array. The unit cell size is $17 \mathrm{~mm} \times 17 \mathrm{~mm}$ corresponding to $0.28 \lambda \times 0.28 \lambda$ where $\lambda$ is free space wavelength at operating frequency $5 \mathrm{GHz}$.

In Fig. 2(a), we plot reflection phase versus outer length $\left(\mathrm{L}_{\mathrm{o}}\right)$ for three different values of inner length i.e. $\mathrm{L}_{\mathrm{i}}$ $=3,5$ and $7 \mathrm{~mm}$, respectively. The graphs in Fig. 2(a) show that phase range is $\sim 275^{\circ}$, which is much lower than the required $360^{\circ}$ range. In Fig. 2(b), we plot sensitivity of three reflection phase graphs. Sensitivity is defined as the partial derivative of phase diagram with respect to relevant geometrical parameter [19].
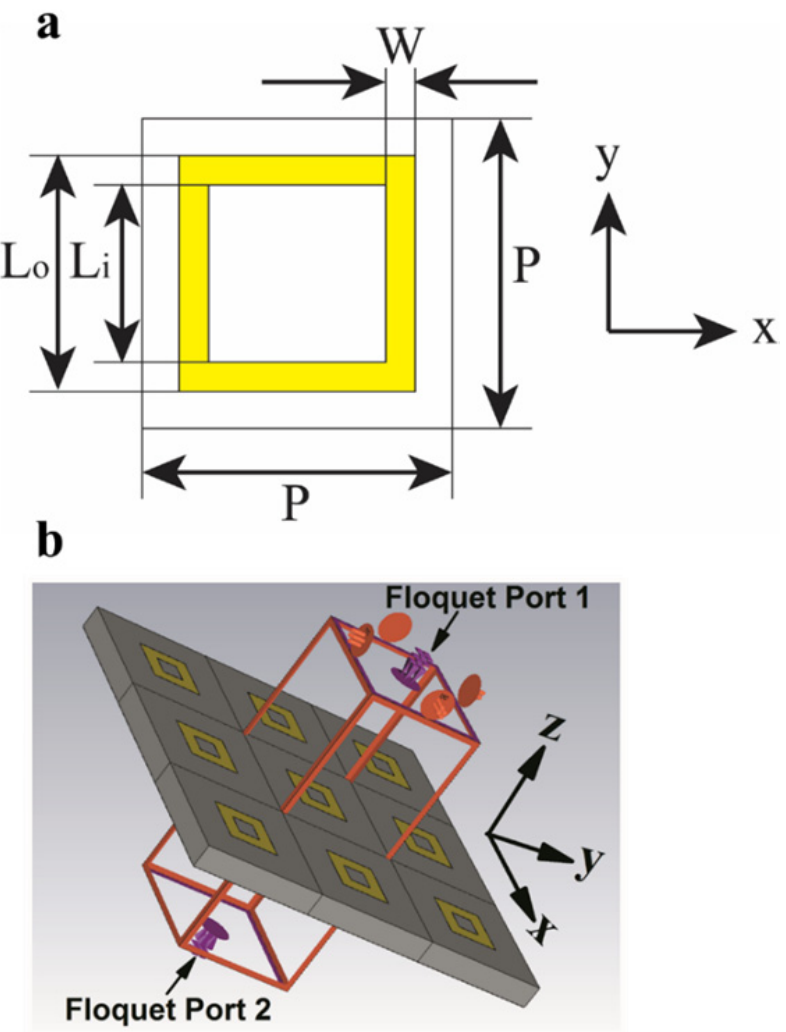

Fig. 1. a) Schematic of single square ring. (b) Boundary conditions for unit cell simulation.

a

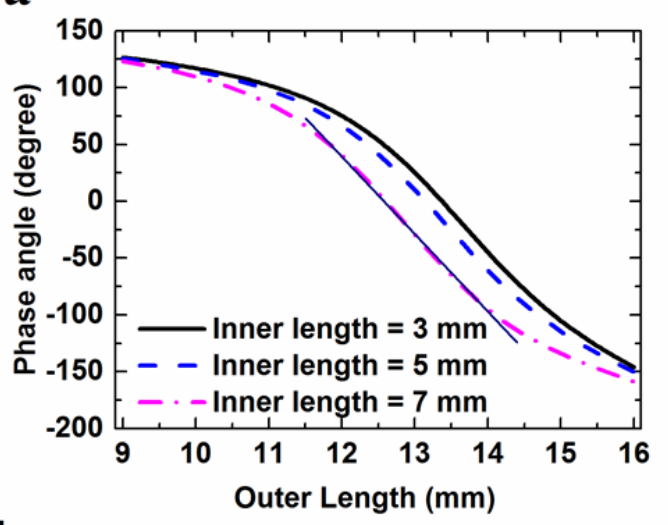

b

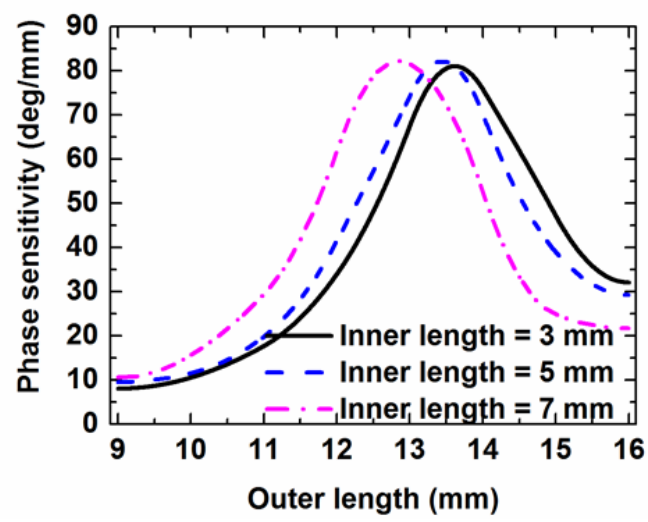

Fig. 2. (a) Reflection phase versus outer length for fixed inner lengths. (b) Phase sensitivities of phase graphs in Fig. 2(a). 
From Fig. 2(b) it is seen that the maximum phase sensitivity can be as high as $\sim 85^{\circ} / \mathrm{mm}$. This is due to the quick transition of reflection phase between $\mathrm{L}_{\mathrm{o}}=12 \mathrm{~mm}$ and $\mathrm{L}_{\mathrm{o}}=14 \mathrm{~mm}$. The maximum phase sensitivity of $85^{\circ} / \mathrm{mm}$ can cause a phase error of $\pm 17^{\circ}$ with $0.4 \mathrm{~mm}$ etching tolerance [11]. The phase error can be further increased with higher etching tolerance.

In order to solve that, we take seven integer values of outer length $\left(\mathrm{L}_{\mathrm{o}}\right)$ from $10 \mathrm{~mm}$ to $16 \mathrm{~mm}$; between every two subsequent values of outer length (i.e. between $\mathrm{L}_{\mathrm{o}}$ and $L_{o}+1$ ), the inner length dimension is varied for fixed $L_{0}$. As reflection phase changes very slowly with $\mathrm{L}_{\mathrm{i}}$, phase value cannot fall quickly in the middle of two successive values. Thus, phase sensitivity is reduced and we get a linear and flat phase response. The whole procedure is graphically shown in Fig. 3(a). Here, we plot reflection phase versus inner length $\left(L_{i}\right)$ for subsequently fixed values of outer length. First, we take the seven reflection phase values correspond to $\mathrm{L}_{\mathrm{o}}=10 \mathrm{~mm}$ to $16 \mathrm{~mm}$ respectively with $\mathrm{L}_{\mathrm{i}}$ fixed at $3 \mathrm{~mm}$ and plot them along a vertical line (black dash-dotted line) which represents $L_{\mathrm{i}}=3 \mathrm{~mm}$. We start inner length variation from $3 \mathrm{~mm}\left(\mathrm{~L}_{\mathrm{i}}=3 \mathrm{~mm}\right)$ while $\mathrm{L}_{\mathrm{o}}$ is fixed at $10 \mathrm{~mm}$. We end this variation at that value of $\mathrm{L}_{\mathrm{i}}$ whose corresponding reflection phase is just prior to the phase value for $L_{i}=3 \mathrm{~mm}$ and $L_{o}=11 \mathrm{~mm}$. After that, we fix $L_{o}$ at $11 \mathrm{~mm}$ and vary $L_{i}$ from $3 \mathrm{~mm}$ up to the value when the corresponding phase is just ahead of phase value for $\mathrm{L}_{\mathrm{i}}$ and $\mathrm{L}_{\mathrm{o}}=3$ and $12 \mathrm{~mm}$, respectively.

We continue this procedure up to $\mathrm{L}_{\mathrm{o}}=16 \mathrm{~mm}$. Therefore, a continuation is maintained among consecutive segments of phase graph. Figure 3(b) shows the overall re-
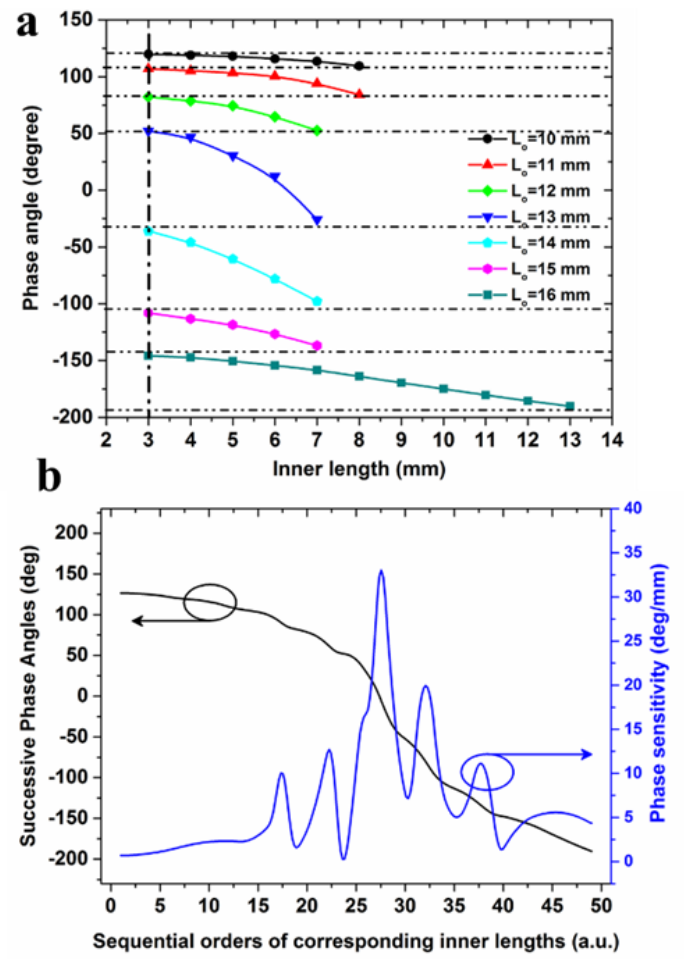

Fig. 3. (a) Reflection phase graphs versus inner length $\left(L_{i}\right)$ for subsequent outer lengths $\left(\mathrm{L}_{\mathrm{o}}\right)$. (b) Overall phase variation versus all successive $\mathrm{L}_{\mathrm{i}}$. flection phase variation as the function of all successive inner lengths. Since reflection phase is now controlled by the inner length dimensions, the phase sensitivity is reduced a lot. The slope of the phase graph plotted in solid blue line shows the maximum phase sensitivity is $34 \% \mathrm{~mm}$. To the best of author's knowledge, it is the lowest phase sensitivity reported to date. Using single square rings $\sim 316^{\circ}$ reflection phase angle from $\sim 126^{\circ}$ to $\sim-190^{\circ}$ is covered.

\subsection{Complementary Square Ring}

The phase range covered by phasing element is defined as follows

Phase range $=$ Final phase - Initial phase .

In the previous section, it is shown that single ring covers the reflection phase ranging from $126^{\circ}$ to $-190^{\circ}$. Therefore, the initial phase is $126^{\circ}$ and final phase is $-190^{\circ}$. As the minimum required phase angle is $360^{\circ}$ to design a reflectarray antenna without introducing any phase error, the current design requires extra reflection phase of at least $\sim 44^{\circ}$. The inadequate phase range is a common issue for designing a reflectarray with subwavelength phasing element [15-17]. This phase range can be covered using a second type of phasing element keeping the grid size same as single square ring. The phase range yet to be attained can be either higher than the initial phase $126^{\circ}$ or lower than the final phase $-190^{\circ}$. We choose complementary

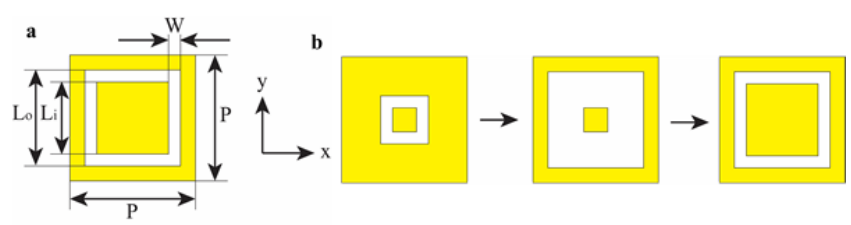

Fig. 4. (a) Schematic of complementary square ring. (b) Outer length variation followed by inner length variation.

$\mathbf{a}$

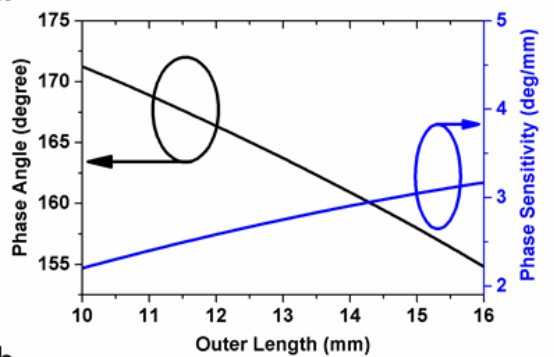

b

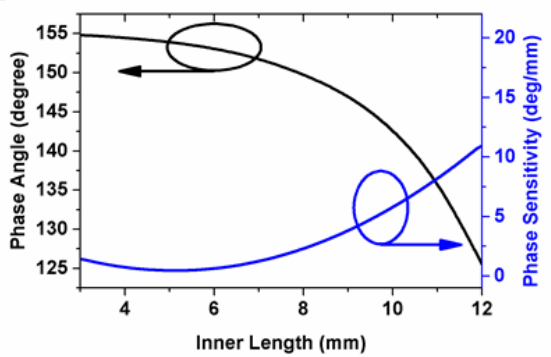

Fig. 5. (a) Reflection phase versus outer length $\left(\mathrm{L}_{\mathrm{o}}\right)$ with inner length fixed. (b) Reflection phase versus inner length with outer length fixed. 
ring as the second type of phasing element, schematically shown in Fig. 4(a), to cover the rest $\sim 44^{\circ}$ phase range, which is higher than $126^{\circ}$.

As shown in Fig. 4(b), to achieve this $44^{\circ}$ phase range first we vary outer length $\left(\mathrm{L}_{0}\right)$ of the complementary ring from $10 \mathrm{~mm}$ to $16 \mathrm{~mm}$ while fixing the inner length $\left(\mathrm{L}_{\mathrm{i}}\right)$ at $3 \mathrm{~mm}$. This covers a phase range from $171^{\circ}$ down to $154^{\circ}$. After that, we fix outer length $\left(\mathrm{L}_{\mathrm{o}}\right)$ at $16 \mathrm{~mm}$ and vary inner length $\left(\mathrm{L}_{\mathrm{i}}\right)$ from $3 \mathrm{~mm}$ to $12 \mathrm{~mm}$. This covers the phase range from $154^{\circ}$ down to $126^{\circ}$. The two reflection phase graphs are shown in Fig. 5 (a) and (b), respectively. The corresponding phase sensitivity graphs are plotted in blue curves that show the phase sensitivity is extremely low for both the graphs.

\section{Full Array Design}

We designed and fabricated a full reflectarray antenna that consisting of 701 phasing elements, with antenna size of diameter $8.5 \lambda$. Simulation of full array is carried out using CST Microwave Studio Integral Equation (IE) Solver. A Matlab Code is written to properly place the appropriate phasing element at different locations of the full array environment according to the required phase compensation. The governing equation to determine the required reflecting phase at $\left(x_{i}, y_{i}\right)$ is given below

$$
\Psi=K_{0}\left[d_{i}-\left\{x_{i} \cos \left(\varphi_{b}\right)+y_{i} \sin \left(\varphi_{b}\right)\right\} \sin \left(\theta_{b}\right)\right]
$$

where $K_{0}(=2 \pi \lambda)$ is the free space propagation constant and $d_{i}$ is the distance between phase center of the feed antenna and the $i$-th unit cell of the array. We consider the array is on x-y plane. $\left(x_{i}, y_{i}, z_{i}\right)$ and $\left(x_{\mathrm{f}}, y_{\mathrm{f}}, z_{\mathrm{f}}\right)$ are the coordinates of the $i$-th unit cell and phase center of feed respectively. $\phi_{\mathrm{b}}$ and $\theta_{0}$ are the azimuthal and elevation angle of the main beam direction respectively, which in this report are chosen to be zero for both. So, main beam would be radiated normally with respect to the plane of reflectarray. A far-field source is selected to illuminate the whole array, which is the radiation pattern of a horn antenna previously simulated in Transient solver in CST MWS. Simulated far-field radiation property of the array is listed in Tab. 1.

The fabricated reflectarray with feeding horn antenna is shown in Fig. 6(a). PTFE (Teflon) substrate of thickness $3.175 \mathrm{~mm}$ is used to fabricate the reflectarray antenna. A pyramidal horn is manufactured to illuminate the array. The phase center of the feeding horn antenna is placed $382.5 \mathrm{~mm}$ in front of the array, which equals to $f / D$ (focus/Diameter) ratio of 0.75 . Figure $7(a)$, (b) show the normalized radiation patterns at $5 \mathrm{GHz}$ for $\mathrm{x}-\mathrm{z}$ and $\mathrm{y}-\mathrm{z}$ planes, respectively. It is evident from Fig. 7 that measured results are well matched with corresponding simulated results.

\begin{tabular}{|c|c|c|c|}
\hline Cut Planes & $\begin{array}{c}\text { Max. Gain } \\
\text { (dB) }\end{array}$ & $\begin{array}{c}\text { 3-dB beam } \\
\text { width }\end{array}$ & SLL (dB) \\
\hline$\Phi=0^{\circ}$ & 26 & $7^{\circ}$ & -16.74 \\
\hline$\Phi=90^{\circ}$ & 26 & $7.3^{\circ}$ & -21.2 \\
\hline
\end{tabular}

Tab. 1. Simulated far-field patterns at two principal planes.
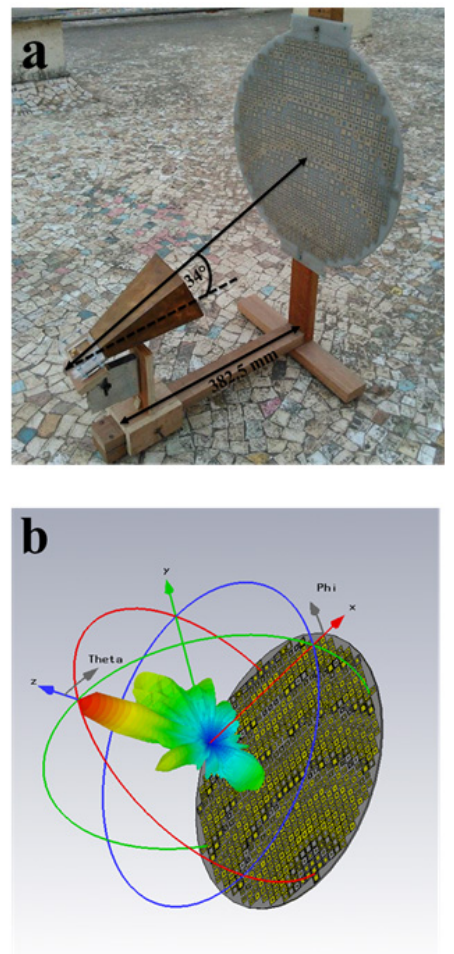

Fig. 6. (a) Photograph of the fabricated reflectarray with feed horn antenna. (b) 3-D simulated radiation pattern.

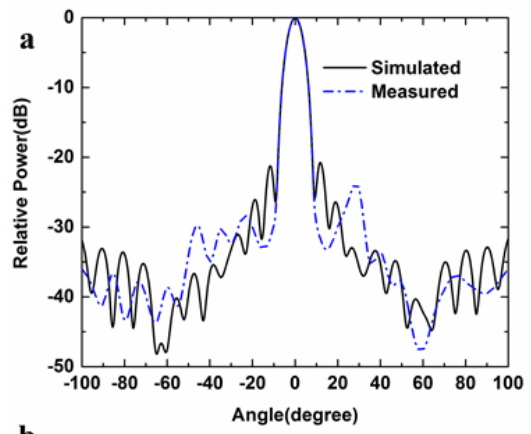

$\mathbf{b}$

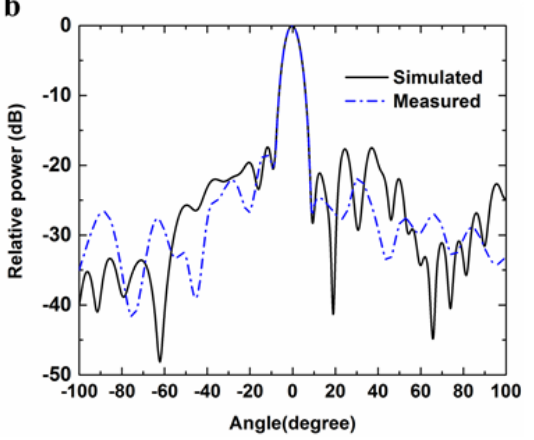

Fig. 7. Simulated and measured co-polarized radiation patterns at $5 \mathrm{GHz}$ : (a) x-z plane $\left(\Phi_{\mathrm{b}}=0^{\circ}\right)$, (b) y-z plane $\left(\Phi_{\mathrm{b}}=90^{\circ}\right)$.

Figure 8(a) shows the electric field distribution in $y-z$ plane at $5 \mathrm{GHz}$. It is seen from the figure that spherical wave front (dashed white line) emitted from the source antenna is converted into a plane wave front (dash dotted white line) after reflected from the reflectarray. Figure 8(b) shows the color mapping of reflection phase for different phasing elements, which confirms the parabolic nature of 
reflection phase distribution. Red and blue color stand for the highest and lowest reflection phase respectively.

Figure 9 plots the measured gain and calculated aperture efficiency versus frequency of the reflectarray. The maximum gain is noticed at $5 \mathrm{GHz}$, which is $26.0 \mathrm{dBi}$. The calculated aperture efficiency $\left(G \lambda^{2} /(4 \pi A)\right.$; where $G, \lambda$ and $A$ stand for gain, wavelength and aperture area, respectively) of the array is $\sim 58 \%$ at $5 \mathrm{GHz}$. After $5 \mathrm{GHz}$, reflectarray gain $G$ slightly decreases up to $6 \mathrm{GHz}$. The wavelength $\lambda$ also decreases as frequency increases. Therefore, aperture efficiency is degraded after $5 \mathrm{GHz}$. The gain of the antenna can be enhanced further by properly optimizing the $f / D$ ratio. Therefore, the aperture efficiency can also be improved. From Fig. 9, it can be seen that the 3-dB gain bandwidth is $\sim 45.4 \%$. Therefore, a broadband performance is achieved. We also plot the normalized radiation patterns at different frequencies from $4.5 \mathrm{GHz}$ to $6.5 \mathrm{GHz}$ in Fig. 10(a) and (b) for $x-z$ and $y-z$ plane, respectively. The similar patterns at different frequencies ensure the broadband nature of the designed reflectarray.

Moreover, we also plot the measured reflection coefficient versus frequency graphs in Fig. 11. Reflection coefficient is the ratio of reflected and incident power measured at the port of an antenna. However, the reflectarray structure itself has no port, as it is illuminated with a feeding horn. Therefore, we measure the reflection coefficient of both horn antenna alone (magenta, dash-dotted curve) and with the reflectarray as well (black, dashed curve). The power already lost at the port of horn antenna cannot reach up to the reflectarray surface. Therefore, the reflection coefficient of reflectarray with horn significantly depends on the reflection coefficient of the horn antenna alone. Thus, we eliminate the reflection coefficient of horn antenna alone from the reflection coefficient of horn with reflectarray. As
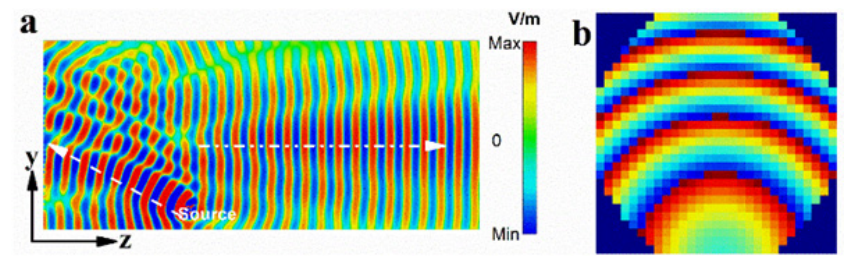

Fig. 8. (a) E-field distribution at $5 \mathrm{GHz}$. (b) Phase distribution of the reflectarray at $5 \mathrm{GHz}$.

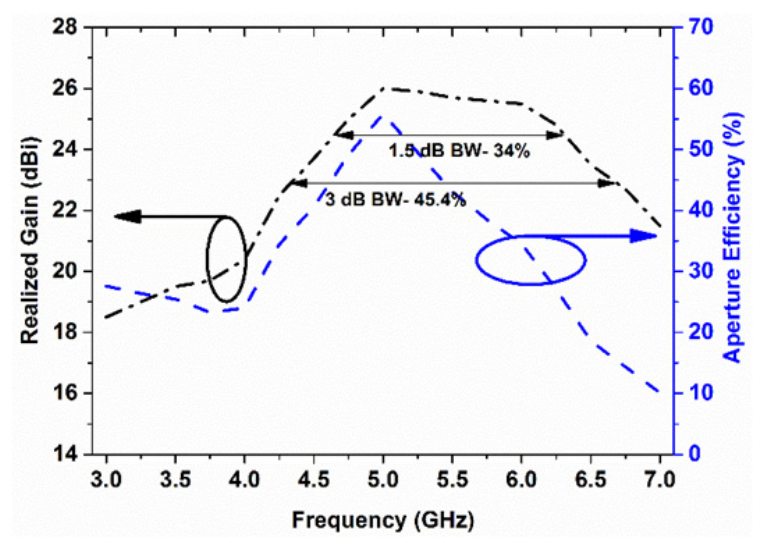

Fig. 9. Realized gain and aperture efficiency vs. frequency.
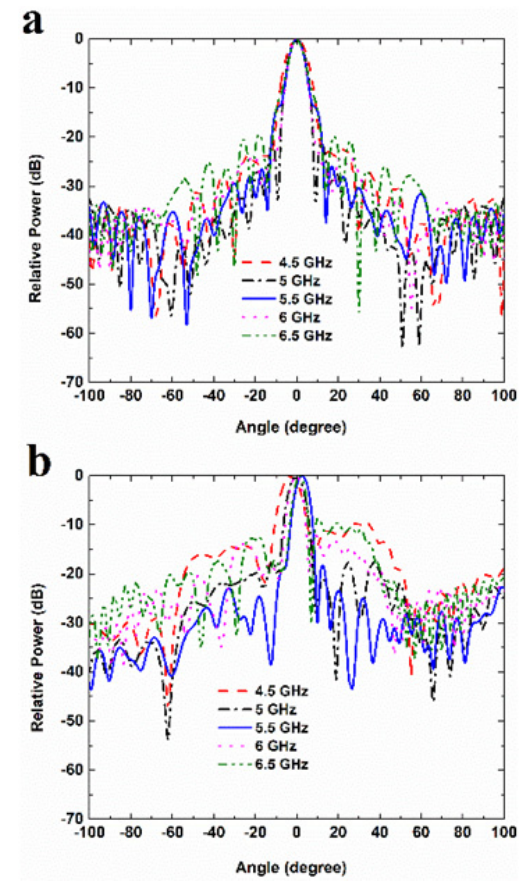

Fig. 10. Normalized radiation patterns at different frequencies (a) x-z plane $\left(\Phi_{\mathrm{b}}=0^{\circ}\right)$, (b) y-z plane $\left(\Phi_{\mathrm{b}}=90^{\circ}\right)$.

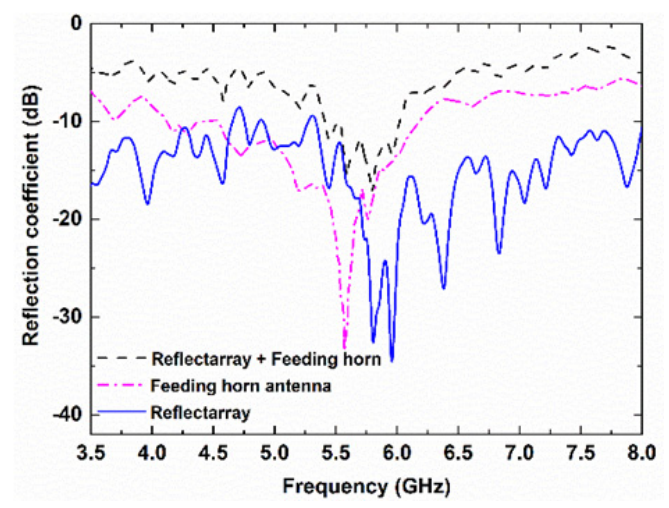

Fig. 11. Reflection coefficient versus frequency.

a result, we get the normalized reflection coefficient at reflectarray surface. We plot the normalized reflection coefficient (solid, blue curve) that is less than $-10 \mathrm{~dB}$ for the entire range from $3.5 \mathrm{GHz}$ to $8 \mathrm{GHz}$. The result implies that the designed reflectarray antenna works well over this range of frequency.

\section{Conclusion}

Combination of two reduced sized phasing elements for a compact reflectarray antenna is proposed. It is shown that varying the inner length of the single ring for successive fixed values of outer length can give a reflection phase graph with the gentler slope. As the full $360^{\circ}$ phase range cannot be attained using the single ring, another set of complementary rings is used to cover the rest of the phase angle. Therefore, to keep phase sensitivity lower, the reflection phase range is not compromised for a phasing element with size $0.28 \lambda \times 0.28 \lambda$. A 701-element reflectarray is designed, fabricated and measured. Measured results 
show good agreement with corresponding simulated results. The achieved $3-\mathrm{dB}$ gain bandwidth is $45.4 \%$, which confirms the broadband behavior of the reflectarray.

\section{Acknowledgments}

We would like to acknowledge the help from $\mathrm{Mr}$. Avik Hati for formulating the algorithm to automatize the full array design and Mr. Jishnu P. during the measurement process.

\section{References}

[1] HUANG, J., ENCINAR, J. A. Reflectarray Antennas. Hoboken (NJ, USA): IEEE Press, 2007. ISBN: 9780470178768

[2] POZAR, D. M., TARGONSKI, S. D., SYRIGOS, H. D. Design of millimeter wave microstrip reflectarray. IEEE Transactions on Antennas and Propagation, 1997, vol. 45, no. 2, p. 286-296. DOI: $10.1109 / 8.560348$

[3] MOUSTAFA, L., GILlARD, R., PERIS, F., et al. The phoenix cell: a new reflectarray cell with large bandwidth and rebirth capabilities. IEEE Antennas and Wireless Propagation Letters, 2011, vol. 10, p. 71-74. DOI: 10.1109/LAWP.2011.2108633

[4] BIALKOWSKI, M. E., SAYIDMARIE, K. H. Investigations into phase characteristics of a single-layer reflectarray employing patch or ring elements of variable size. IEEE Transactions on Antennas and Propagation, 2008, vol. 56, no. 11, p. 3366-3372. DOI: 10.1109/TAP.2008.2005470

[5] RAJAGOPALAN, H., SHENHENG XU, RAHMAT-SAMII, Y. On understanding the radiation mechanism of reflectarray antennas: An insightful and illustrative approach. IEEE Antennas and Propagation Magazine, 2012, vol. 54, no. 5, p. 14-38. DOI: 10.1109/MAP.2012.6348112

[6] ENCINAR, J. A. Design of two-layer printed reflectarrays using patches of variable size. IEEE Transactions on Antennas and Propagation, 2001, vol. 49, no. 10, p. 1403-1410. DOI: $10.1109 / 8.954929$

[7] HAMZAVI-ZARGHANI, Z., ATLASBAF, Z. A new broadband single-layer dual-band reflectarray antenna in $\mathrm{X}$ - and Ku-bands. IEEE Antennas and Wireless Propagation Letters, 2014, vol. 14, p. 602-605. DOI: 10.1109/LAWP.2014.2374351

[8] HAN, C., ZHANG, Y., YANG, Q. A broadband reflectarray antenna using triple gapped rings with attached phase-delay lines. IEEE Transactions on Antennas and Propagation, 2017, vol. 65, no. 5, p. 2713-2717. DOI: 10.1109/TAP.2017.2679493

[9] TIAN, C., JIAO, Y. C., LIANG, W. L. A broadband reflectarray using phoenix unit cell. Progress In Electromagnetics Research Letters, 2014, vol. 50, p. 67-72. DOI: 10.2528/PIERL14093003

[10] LEE, S. R., LIM, E. H., LO, F. L. Broadband single-layer E-patch reflectarray. Radioengineering, 2017, vol. 26, no. 1, p. 97-106. DOI: $10.13164 /$ re.2017.0097

[11] DENG, R., XU, S., YANG, F. Design of a Ku/Ka quad-band reflectarray antenna for satellite communications. In IEEE International Symposium on Antennas and Propagation. Fajardo (Puerto Rico), 2016. DOI: 10.1109/APS.2016.7696316

[12] YOON, J. H., YOON, Y. J., LEE, W., et al. Square ring element reflectarrays with improved radiation characteristics by reducing reflection phase sensitivity. IEEE Transactions on Antennas and Propagation, 2015, vol. 63, no. 2, p. 814-818. DOI: 10.1109/TAP.2014.2379919
[13] CADORET, D., MARNAT, R., LOISON, R., et al. A dual linear polarized printed reflectarray using slot loaded patch elements. In The Second European Conference on Antennas and Propagation. Edinburgh (UK), 2007. DOI: 10.1049/ic.2007.1620

[14] CAI, T., WANG, G. M., ZHANG, X. F. Compact dual-resonance element with low phase sensitivity for offset reflectarray antennas. IEEE Antennas and Wireless Propagation Letters, 2016, vol. 16, p. 1213-1216. DOI: 10.1109/LAWP.2016.2628584

[15] POZAR, D. M. Wideband reflectarrays using artificial impedance surfaces. Electronics Letters, 2007, vol. 43, no. 3, p. 148-149. DOI: $10.1049 / \mathrm{el}: 20073560$

[16] ZHAO, G., JIAO, Y. C., ZHANG, F., et al. A subwavelength element for broadband circularly polarized reflectarrays. IEEE Antennas and Wireless Propagation Letters, 2010, vol. 9, p. 330 to 333. DOI: 10.1109/LAWP.2010.2047836

[17] NAYERI, P., YANG, F., ELSHERBENI, A. Z. Bandwidth improvement of reflectarray antennas using closely spaced elements. Progress In Electromagnetics Research C, 2011, vol. 18, p. 19-29. DOI: 10.2528/PIERC10091505

[18] PATtanAYAK, A., RANA, G., DUTtaguPTA, S. P., et al. A broadband reflectarray with combination of subwavelength phasing elements. In Asia-Pacific Microwave Conference. New Delhi (India), Dec. 2016. DOI: 10.1109/APMC.2016.7931462

[19] BOZZI, M., GERMANI, S., PERREGRINI, L. Performance comparison of different element shapes used in printed reflectarrays. IEEE Antennas and Wireless Propagation Letters, 2003, vol. 2, p. 219-222. DOI: 10.1109/LAWP.2003.819687

\section{About the Authors ...}

A. PATTANAYAK (pursuing Ph.D. in IIT Bombay) focuses his research on antenna and metasurface design for microwave and TeraHertz frequency range.

G. RANA (pursuing Ph.D. in IIT Bombay) obtained his B.E. and M.E. from the Bengal Engineering and Science University, Shibpur, West Bengal, India in 2009 and 2012, respectively. His research focuses on Microwave and $\mathrm{THz}$ plasmonic devices and $\mathrm{THz}$ photo-conducive antenna.

S. P. DUTTAGUPTA obtained B.Tech. (Honours) in Electronics from IIT Kharagpur, India, and Ph.D. from the University of Rochester, New York, USA. Currently, he is an associate professor in the Electrical Engineering Dept. at IIT Bombay. His research effort focuses on highly directive antenna design, TeraHertz spectroscopy and micro, nano fabricated devices.

P. S. GANDHI received the B.Eng. degree from the University of Bombay, India, the M.Tech degree from IIT Bombay, Mumbai; and the Ph.D. degree from Rice University, Houston, TX, USA, all in mechanical engineering. Currently, he is a professor in the Mechanical Engineering Dept. at IIT Bombay. His research effort focuses on mechatronics and microelectromechanical systems.

A. A. DESHMUKH obtained B.E. in Electronics from V.I.T. Pune University, India, M.Tech. degree in 2000 and Ph.D. in 2004 from the Dept. of Electrical Engineering, IIT Bombay. Currently, he is working as Professor and Head of EXTC Dept. at D. J. Sanghvi College of Engineering, Mumbai. His research effort focuses on microstrip antenna design. 